\title{
Effect of Dark Adaptation and Bleaching on Blue Light Reflectance Imaging in Macular Telangiectasia Type 2
}

\author{
Mali Okada, MBBS, MMed,* Tjebo F.C. Heeren, MD,*† Catherine A. Egan, MBBS, \\ FRANZCO,* Vincent Rocco,* Roberto Bonelli, M.Sc, $\$ \S$ Marcus Fruttiger, PhDJ
}

* Moorfields Eye Hospital NHS Foundation Trust, London, UK

† Department of Ophthalmology, University of Bonn, Bonn, Germany

\$Walter and Eliza Hall Institute of Medical Research, Melbourne, Australia

$\S$ The University of Melbourne, Melbourne, Australia

I UCL Institute of Ophthalmology, London, United Kingdom

\section{Short title:}

Blue Light Reflectance in MacTel Type 2

\section{Funding:}

Supported by the Lowy Medical Research Institute, Sydney, Australia as part of The Macular Telangiectasia Project. This work was also made possible through the Victorian State Government Operational Infrastructure Support and Australian Government National Health and Medical Research Council (NHMRC) Independent Research Institute Infrastructure Support Scheme (IRIISS). R.B. was supported by the Melbourne International Research Scholarship. No funding was received from the National Institutes of Health (NIH), the Wellcome Trust or the Howard Hughes Medical Institute (HHMI).

\section{Financial disclosures:}

None

\section{Address for correspondence:}

Marcus Fruttiger, PhD, University College London, UCL Institute of Ophthalmology, London EC1V 9EL, United Kingdom.m.fruttiger@ucl.ac.uk 


\section{Key words}

Macular telangiectasia type 2 (MacTel); confocal blue light reflectance (BLR); scanning laser ophthalmoscopy; diagnostic imaging; photopigment; photobleaching; dark adaptation; macular pigment

\section{Summary Statement}

The macular telangiectasia type 2 characteristic changes on blue light reflectance imaging are affected by photobleaching. 


\begin{abstract}
Purpose

In patients with macular telangiectasia (MacTel) type 2, blue light reflectance (BLR) imaging reveals an oval, parafoveal area in the macula that has increased reflectance compared to its surround. Here we examine how dark adaptation and photobleaching can affect the BLR imaging pattern.
\end{abstract}

\title{
Methods
}

Prospective study of patients with MacTel enrolled in the MacTel Natural History Observation Study. After dark-adaption, a sequence of images was obtained with a confocal scanning laser ophthalmoscope at $488 \mathrm{~nm}$. Change of reflectance patterns was analyzed over time.

\section{Results}

Eighteen eyes from 16 patients were analyzed. Initially, increased reflectivity in the parafoveal area resulted in higher gray values compared to the paramacular surround on BLR imaging. The difference between parafoveal and paramacular reflectance intensity decreased steadily during imaging, from 17.7 gray-value units (95\% Confidence Interval [CI]: 12.1-23.2) down to 2.8 (95\% CI: $-0.8-6.5)$ after around 30 seconds, and recovered after 5 minutes of dark adaptation.

\section{Conclusion}

A bleaching effect was evident in our study. Understanding these changes is important for both diagnosis and assessment of BLR phenotype in patients with MacTel and could also provide further insights into the pathophysiology of this disease. 


\section{Introduction}

Macular telangiectasia (MacTel) type 2 is a macular disorder characterised by progressive neurodegeneration with associated vascular changes. ${ }^{1}$ Since the original classification by Gass and Blodi ${ }^{2}$ there has been significant change in our understanding of this disease. The advent of modern imaging techniques, including optical coherence tomography (OCT), has aided not only in phenotyping the condition beyond the previous gold standard ophthalmoscopy and fluorescein angiography features, but also added to the information derived from recent histological data. ${ }^{1,3-6}$

In particular, blue light reflectance (BLR) imaging using confocal scanning laser ophthalmoscopy (SLO) at a wavelength of $488 \mathrm{~nm}$, is promising as a rapid, non-invasive diagnostic tool for MacTel type 2. It shows a highly characteristic oval parafoveal area of increased reflectance and is often surrounded by a rim of lower reflectance. ${ }^{7,8}$ The area of increased reflectance is consistently larger than the area of hyperfluorescence seen in late phase fluorescein angiography and correlates well with the zone of macular pigment loss, pathognomonic for this disease. ${ }^{7,9}$ Therefore, loss of macular pigment has been proposed to cause the observation seen on BLR imaging. However, the exact basis for this pattern is still unclear.

Although the pattern on BLR imaging is highly characteristic for MacTel type 2, there can be significant inter- and even intra-individual variation in the observed extent and intensity of increased reflectance compared to the background. In the original study reported by Charbel Issa et al, enhanced parafoveal reflectance was not evident in 4 of 66 eyes: two of whom had very early disease, one eye with intraretinal pigment clumping (although most with pigment clumping had increased reflectance) and another with 
previous macular hole surgery. ${ }^{7}$ Although this represents only a small proportion of their cohort, it could potentially lead to equivocal diagnosis if the BLR imaging is interpreted in isolation.

In clinical practice we have observed that the increased parafoveal BLR appears more prominent when imaging is performed before rather than immediately after other imaging modalities. Recently, it has been reported that the parafoveal graying seen on fundus examination in patients with MacTel type 2 diminished with continuous light exposure and returned once dark adapted. ${ }^{10}$ As we anticipated a similar effect in BLR imaging, we investigated the effect of dark adaptation and subsequent retinal bleaching on the observed BLR pattern in order to improve imaging protocols as well as gain insights into the pathophysiology of MacTel.

\section{Materials and Methods}

\section{Study population}

This was a prospective observational study of patients with a diagnosis of MacTel type 2 who were participants of the international MacTel Natural History Observation Study. The detailed protocol has been published previously. ${ }^{11,12}$ All patients had standard BLR imaging as part of their enrolment into the registry. Control participants were recruited for comparative purposes. The study was approved by the local institutional review board and conducted according to the principles of the Declaration of Helsinki. A written, informed consent was obtained from all participants.

\section{BLR imaging}


BLR imaging was acquired using the Heidelberg Spectralis ${ }^{\circledR}$ SLO system (Heidelberg Engineering, Heidelberg, Germany) with an excitation wavelength of 488nm. Pupils were dilated using both tropicamide $1 \%$ and phenylephrine $2.5 \%$ eye drops for 30 minutes. Image alignment and focus was optimized before patching both eyes of the patient to allow for 15 minutes dark adaptation. Once the eye patch was removed, imaging immediately commenced using blue light with a sequence of 15 images with an interval of around 2 seconds between each image. Eyes were then allowed to re-dark adapt for a shorter 5 minute period before a further image was taken. The gain on the non-normalized images was not adjusted throughout the entire image acquisition period and the same photographer performed all imaging.

In order to compare the effect of photobleaching within the same image, a separate group of patients underwent split imaging. This cohort completed the same dark adaptation process as described, but was then asked to look at a superior fixation target at the time of imaging with the blue laser illumination directed over the superior hemi-retina. After 15 images, the patient was then directed to fixate centrally and a further image was taken, now with illumination over the entire posterior pole. This last image thus presented a combined image with the superior half subjected to the effects of bleaching and the inferior half of the image comparatively less so.

\section{Image Analysis}

To ensure consistency of analysis across the sequence of 15 images for each patient, images were imported into Adobe Photoshop CS5 software (Adobe Systems Inc. San Jose, California) and auto-alignment performed based on retinal features to remove any differences due to eye movement. Objective quantification of the intensity of the 
parafoveal reflectance and surrounding background was obtained using conventional 8 bit grayscale values in Image J software (ImageJ version $1.50 \mathrm{i}$ available at https://imagej.nih.gov/ij/index.html) with a linear scale from 0 (black) to 255 (white). For each image, the mean brightness of the pathological parafoveal reflectance was analysed and compared to the surrounding macular region (Figure 1). The gray level difference between the two region of interest rather than the absolute value of each was used as the primary measure. This was to offset any artefactual variation in the fundus illumination over time such as from tilt or pupil size and any differences in detector settings or fundus reflectivity between patients. In the control participants, the template region of interest used for the MacTel eyes was used to demarcate the parafoveal and paramacular areas.

All statistical analyses were performed using the Statistical Package for the Social Sciences v.20 (IBM SPSS Inc, Chicago IL) and R statistical software (R development Core Team, Vienna Austria). A linear mixed regression model was used to analyse the trend over time with the $\mathrm{p}$-value derived from T-statistics. A p value $<0.05$ was considered the statistical level of significance. Results were also analysed to look for correlation between eyes when both eyes from a single patient was included.

\section{Grading}

The BLR images were graded on a scale from 0 to 3 with increasing areas of parafoveal increased reflectance - Table 1. Baseline images after dark adaptation were graded and compared to the last images in the sequence. A second masked grader repeated the image grading and a kappa value calculated for inter-rater agreement. 


\section{Results}

\section{Patient characteristics}

Twenty-two eyes from 20 patients were included in the study; 18 eyes from 16 patients had standard imaging and an additional 4 eyes from 4 patients had split imaging performed. Mean age was 55.6 years (range $41-78$ years) with approximately half being female $(55 \%, n=11)$. The majority of patients were of Caucasian ethnicity $(75 \%, n=15)$. Four eyes had intraretinal pigment clumping and none had secondary subretinal neovascular membrane. Five control eyes also underwent dark adaptation and had the same sequence of imaging performed for comparison.

\section{Effect of dark adaptation and bleaching}

Continuous imaging of MacTel patients in the BLR imaging mode for approximately 30 seconds noticeably degraded the pattern seen in the first exposure, with an apparent loss of contrast between the (brighter) parafoveal and the (darker) surrounding paramacular area (Figure 2). Quantification of gray values in the image sequences from 18 eyes revealed a decreased difference between the two areas from 17.7 gray value units (SE: 2.6, $95 \%$ CI: $12.1-23.2)$ immediately after dark adaptation down to 2.8 gray value units (SE: $1.7,95 \%$ CI: $-0.8-6.5$ ) by the end of the bleaching sequence (diamond data points in Figure 3). In contrast, in healthy controls the parafoveal area was darker than the surrounding paramacular area (leading to negative difference values in Figure 3), presumably due to macular pigment in the centre. Furthermore, there was no statistically significant change in the difference between parafoveal and the surrounding paramacular reflectance values in the control patients over time from -12.47 gray value units (SE: 2.07, 95\% CI: -18.2 to -6.73 ) to -19.72 gray value units (SE: 3.67, 95\% CI: -29.9 to -9.55 ) after bleaching (circle data points in Figure 3). 
With linear mixed regression modelling, there was a significant difference in the difference between parafoveal and the surrounding paramacular reflectance values between patients versus controls (model effect 31.39 gray units, $\mathrm{p}<0.001$ ), consistent with the characteristic increased reflectance seen in MacTel. A bleaching effect was evident in patients but not controls in the mixed model, with time a significant factor only when taking into account disease status - Table 2 .

In the sub-group analysis of MacTel patients alone, the effect magnitude of time (model effect -1.07 gray units per image time, $\mathrm{p}<0.05$ ) was even greater compared to the overall model (effect -0.85 gray units per image time, $p=0.04$ ). This is due to the exclusion of healthy controls in the sub-group analysis, which do not show a bleaching effect, diluting the effect magnitude in the overall model. In MacTel patients, the bleaching phenomenon appeared to plateau after 10 images (Figure 3). The slope declined from -1.47 gray units per image in the first 9 images down to -0.58 gray units per image from image 10 onwards. There was no difference between right and left eyes in either model.

Upon re-dark adaptation for 5 minutes, there was evidence of recovery from bleaching and the contrast in reflectance between the parafoveal MacTel area and the paramacular area returned with a mean 14.6 gray unit difference (SE 3.40, 95\% CI: $3.72-25.48$ ), which is similar to the difference initially observed before the photobleaching (17.7 gray units).

In some patients we also performed split imaging, whereby using a superior fixation target only the superior half of the retina was photobleached (over around 30 second). For 
the final image, the fixation target was moved to the centre, demonstrating a brighter superior hemi-retina (corresponding to the bleached area) and a relatively non-bleached darker inferior hemi-retina (see Figure 4). The reflectance intensity difference was most pronounced at the temporal macula region with a clearly visible horizontal demarcation line in some eyes. Analysis of the difference in reflectance intensity across this meridian showed the bleached superior paramacular area was a mean 16.8 gray value units brighter (SE 3.10, 95\% CI: 6.9 - 26.6) compared to the inferior half. This difference corresponds well with the change between parafoveal and paramacular reflectance intensities seen over time in the standard patient group (14.9 gray value units).

In some eyes that demonstrated marked effects of bleaching, the changes to the parafoveal reflectance pattern did not occur uniformly. At baseline, the reflectance was evenly increased, but over time became darker centrally with preservation of a ring of increased reflectance only at the outer border of the MacTel region (arrow in Figure 5). These changes were most evident in the patients who had Grade 3 complete parafoveal area of increased reflectance type morphology.

\section{Grading of BLR patterns}

In order to establish how photobleaching may influence the grading of BLR patterns (Table 1), images were graded by two graders before and after photobleaching. At baseline after dark adaptation (before bleaching), increased parafoveal reflectance was clearly evident in all patients and there was good agreement between the two graders with $\kappa=0.65(95 \%$ CI $0.47-0.83, \mathrm{p}<0.05)$. After bleaching, five of the 18 eyes had a reduction in their grading by at least one level as agreed by both graders (one by 3 levels, one by 2 levels and three by 1 level). In one patient, who had early disease with only 
temporal wedge involvement on the dark adapted image, the bleached image had minimal parafoveal reflectance and if viewed alone, was borderline for diagnosis of MacTel type 2.

\section{Discussion}

In this study, we demonstrate that the increased parafoveal reflectance in BLR imaging becomes less obvious with photobleaching. This has important implications for clinical diagnosis and image grading. Furthermore, understanding the mechanisms responsible for this phenomenon might provide further insight into MacTel pathology.

It is likely that the BLR pattern is caused by the localised lack of macular pigment in the parafoveal regions in MacTel patients. However, Lutein and Zeaxanthin, to our knowledge, should not be affected by photobleaching. This then raises the question how the increased reflectance signal can become less obvious with bleaching.

Our study shows that this effect may be partly due to "lightening up" of the surrounding paramacula. With continued blue light exposure, the absorption of light in the normal, surrounding paramacula declines as 11-cis-retinal in rod outer segments is not sufficiently fast replenished and becomes depleted. This leads to decreased light absorption (i.e. increased reflectance as demonstrated in the patients who had split imaging performed) and results in a reduction of visible contrast between the parafoveal MacTel area and its surrounds. The diseased area does not seem to undergo visual pigment photobleaching to the same degree, hence the loss of contrast. It might be expected that some contrast would be retained between the parafoveal area and the immediate paramacular surrounds due to the preserved ring of macular pigment at the outer border of the MacTel area. 
Interestingly, we did not see that here and a closer approximation between the two areas' gray value was observed. This could be due the width of the paramacular area analysed, which would partially include a band of retina more eccentric to the preserved ring of macular pigment. In healthy eyes, there is reduced macular pigment with increasing eccentricity, and the inclusion of this more eccentric area could have diluted the overall differences in grey values. Visually however, the border between the two areas did appear less distinct with bleaching. Of note, the bleaching induced reduction in gray value differences (green data points in figure 3) was very similar in magnitude to the differences found in the split imaging approach (14.9 versus 16.8), suggesting that changes in the paramacular surround can largely explain the loss of apparent contrast caused by the photobleaching. In healthy controls a bleaching effect also occurs, but as it is applied uniformly to both the parafoveal and paramacular area, the magnitude of this difference remains consistent over time.

However, another contribution to the bleaching phenomenon in MacTel may be due to changes that occur within the perifovea itself. Indeed, in some patients, there was some evidence of a relative darkening within the perifoveal region, with apparent preservation of the intensity of reflectance at the outer border of the MacTel region (Fig. 5). The basis for this may relate to the property of Müller cells as an optical conduit for transmitting light (as demonstrated in animal models) ${ }^{13}$ or due to delayed recycling of photopigment; but why this perifoveal change is only observed in the subset of patients with more extensive areas of reflectance is unclear. Although this outer ring phenotype has been previously described, ${ }^{7}$ the development of this appearance with bleaching has not been reported until now. 
The phenomenon of photobleaching-induced changes in the BLR pattern in MacTel patients might be of similar origin as the decrease of parafoveal graying during clinical examination with an indirect ophthalmoscope, previously described by Jindal et al. ${ }^{10}$ After a further 15-minute dark adaptation period, the gray appearance returned. The authors speculated this could be as a result of dysfunction of Müller cells, which is involved in normal recycling of 11-cis-retinoids. However, the fundoscopic graying observed often diminishes with disease progression, which would not be expected if this phenomenon were due to reduction in macular pigment and/or photopigment alone. An alternate mechanism for the fundoscopic graying may arise from increased light scatter from disorganised or oedematous retina and the reduction with disease severity a consequence of reduction in this backscatter with neuroretinal thinning. However, the physiologic basis for this fundoscopic graying is likely complex and is currently not well understood.

A caveat is that currently, the BLR imaging modality in the confocal SLO (Heidelberg Spectralis ${ }^{\circledR}$ SLO system) used in this study is based on a mix of fundus autofluorescence and BLR and does not measure pure reflectance alone. A bleaching effect has been well described in autofluorescence imaging modality and is likely to play a role here. ${ }^{14-16}$ Theelen and co-authors demonstrated a similarly increased signal and saturation plateau using $488 \mathrm{~nm}$ excitation wavelength on autofluorescence due to absorption by macular pigment. ${ }^{14}$ Furthermore, previous studies on quantitative autofluorescence have also shown that with a confocal SLO, the photobleaching effect is complete by around 20 seconds in rods and slightly longer at 30 seconds in foveal cones with blue wavelength exposure. ${ }^{17,18}$ Although we did not time our imaging sequence in this study, each image was taken with an approximate 2 second interval so the plateau effect of differences in 
reflectance intensity we observed after 10 images in our cohort is consistent with this 2030 second bleaching period.

Aside from understanding how the bleaching phenomenon develops in relation to the MacTel pathophysiology, the results also pose important considerations for clinical practice. Firstly, we suggest that BLR imaging should be performed as the initial imaging modality when a sequence of multimodal imaging for MacTel type 2 is performed for diagnostic purposes. This is particularly valuable in patients with early disease or asymmetric cases, where the other eye may be presumed normal or have minimal OCT features. If there is any doubt with this first image as to the presence or extent of the parafoveal hyper-reflectance, then a short period of dark adaptation may help enhance it. Although our protocol involved initial dark adaptation for 15 minutes, the 5-minute readaptation demonstrated recovery from the bleaching effects and this shorter period may be adequate in a busy real world context.

Secondly, awareness of the bleaching effect is also important in standardizing assessment and classification of the BLR images for research purposes. Our study might also help to establish protocols for imaging studies to obtain maximum and reproducible BLR reflectance.

There are limitations to this study. Although our results were highly significant with very small p-values, this was a small study and the findings may not necessarily be generalizable to all phenotypes of MacTel type 2. We did however attempt to include a broad spectrum of patients from early to advanced disease. In addition, BLR imaging depends on operator technique and is subject to artefacts, eg. variable retinal illumination 
of the retina due to change in angle, tilt, pupil dilation or starting gain on the imaging system. In order to account for this, analysis was done of the difference between the parafoveal and paramacular reflectance intensities within the same image, rather than raw values across the sequence of images. We also investigated for the effects of tilt by changing the camera alignment and found the bleaching phenomenon to remain consistent even in different positions (data not shown). Also, in some eyes, there was an inevitable time lag to capture the first image in order to focus, resulting in partial bleaching of the baseline image, which could lead to an underestimation of the bleaching effect. We are confident that these methodological problems are negligible as we find a significant effect overall and also within individual results.

\section{Conclusion}

This study provides new insight into the mechanism of the blue light reflectance phenomenon in MacTel type 2 by assessing the effect of dark adaptation and bleaching. Our results indicate that the contrast between the parafoveal reflectance and the paramacular surrounds decrease over time predominantly from the effects of bleaching in the surrounding area but also from changes within the MacTel area itself. It also demonstrates the clinical utility of a short period of dark adaptation in enhancing the parafoveal reflectance not only for diagnostic but also for grading and follow-up purposes. 


\section{References}

1. Charbel Issa P, Gillies MC, Chew EY, et al. Macular telangiectasia type 2. Prog Retin Eye Res. 2013;34:49-77. doi:10.1016/j.preteyeres.2012.11.002.

2. Gass JD, Blodi BA. Idiopathic juxtafoveolar retinal telangiectasis. Update of classification and follow-up study. Ophthalmology. 1993;100(10):1536-1546.

3. Wu L, Evans T, Arevalo JF. Idiopathic macular telangiectasia type 2 (idiopathic juxtafoveolar retinal telangiectasis type 2A, Mac Tel 2). Surv Ophthalmol. 2013;58(6):536-559. doi:10.1016/j.survophthal.2012.11.007.

4. Powner MB, Gillies MC, Zhu M, Vevis K, Hunyor AP, Fruttiger M. Loss of Muller's cells and photoreceptors in macular telangiectasia type 2. Ophthalmology. 2013;120(11):2344-2352. doi:10.1016/j.ophtha.2013.04.013.

5. Ooto S, Hangai M, Takayama $\mathrm{K}$, et al. High-resolution photoreceptor imaging in idiopathic macular telangiectasia type 2 using adaptive optics scanning laser ophthalmoscopy. Invest Ophthalmol Vis Sci. 2011;52(8):5541-5550. doi:10.1167/iovs.11-7251.

6. Charbel Issa P, Heeren TFC, Kupitz EH, Holz FG, Berendschot TTJM. Very early disease manifestations of macular telangiectasia type 2. Retina Phila Pa. 2016;36(3):524-534. doi:10.1097/IAE.0000000000000863.

7. Charbel Issa P, Berendschot TTJM, Staurenghi G, Holz FG, Scholl HPN. Confocal blue reflectance imaging in type 2 idiopathic macular telangiectasia. Invest Ophthalmol Vis Sci. 2008;49(3):1172-1177. doi:10.1167/iovs.07-0636.

8. Charbel Issa P, Finger RP, Helb H-M, Holz FG, Scholl HPN. A new diagnostic approach in patients with type 2 macular telangiectasia: confocal reflectance imaging. Acta Ophthalmol (Copenh). 2008;86(4):464-465. doi:10.1111/j.16000420.2007.01041.x.

9. Charbel Issa P, van der Veen RLP, Stijfs A, Holz FG, Scholl HPN, Berendschot TTJM. Quantification of reduced macular pigment optical density in the central retina in macular telangiectasia type 2. Exp Eye Res. 2009;89(1):25-31.

doi:10.1016/j.exer.2009.02.006.

10. Jindal A, Choudhury H, Pathengay A, Flynn HWJ. A novel clinical sign in macular telangiectasia type 2. Ophthalmic Surg Lasers Imaging Retina. 2015;46(1):134-136. doi:10.3928/23258160-20150101-26.

11. Clemons TE, Gillies MC, Chew EY, et al. Baseline characteristics of participants in the natural history study of macular telangiectasia (MacTel) MacTel Project Report No. 2. Ophthalmic Epidemiol. 2010;17(1):66-73. doi:10.3109/09286580903450361.

12. Sallo FB, Leung I, Clemons TE, Peto T, Bird AC, Pauleikhoff D. Multimodal imaging in type 2 idiopathic macular telangiectasia. Retina Phila Pa. 2015;35(4):742-749. doi:10.1097/IAE.0000000000000365. 
13. Franze K, Grosche J, Skatchkov SN, et al. Muller cells are living optical fibers in the vertebrate retina. Proc Natl Acad Sci U S A. 2007;104(20):8287-8292. doi:10.1073/pnas.0611180104.

14. Theelen T, Berendschot TTJM, Boon CJF, Hoyng CB, Klevering BJ. Analysis of visual pigment by fundus autofluorescence. Exp Eye Res. 2008;86(2):296-304. doi:10.1016/j.exer.2007.10.022.

15. Choi K-E, Yun C, Kim Y-H, Kim S-W, Oh J, Huh K. The effect of photopigment bleaching on fundus autofluorescence in acute central serous chorioretinopathy. Retina Phila Pa. July 2016. doi:10.1097/IAE.0000000000001170.

16. Staurenghi G, Luiselli C, Levi G, Viola F, Delori F. Retinal photopigment density measured using autofluorescence imaging. Invest Ophthalmol Vis Sci.

2004;45(13):2795-2795.

17. Morgan JIW, Pugh EN. Scanning Laser Ophthalmoscope Measurement of Local Fundus Reflectance and Autofluorescence Changes Arising from Rhodopsin Bleaching and Regeneration. Invest Ophthalmol Vis Sci. 2013;54(3):2048-2059. doi:10.1167/iovs.12-11089.

18. Delori F, Greenberg JP, Woods RL, et al. Quantitative Measurements of Autofluorescence with the Scanning Laser Ophthalmoscope. Invest Ophthalmol Vis Sci. 2011;52(13):9379-9390. doi:10.1167/iovs.11-8319. 


\section{Figures}

Figure 1.

Image analysis in Image J software comparing difference in reflectance signals between parafoveal (1) and paramacular (2) area. Overlay denotes region of interest for calculation of gray values.

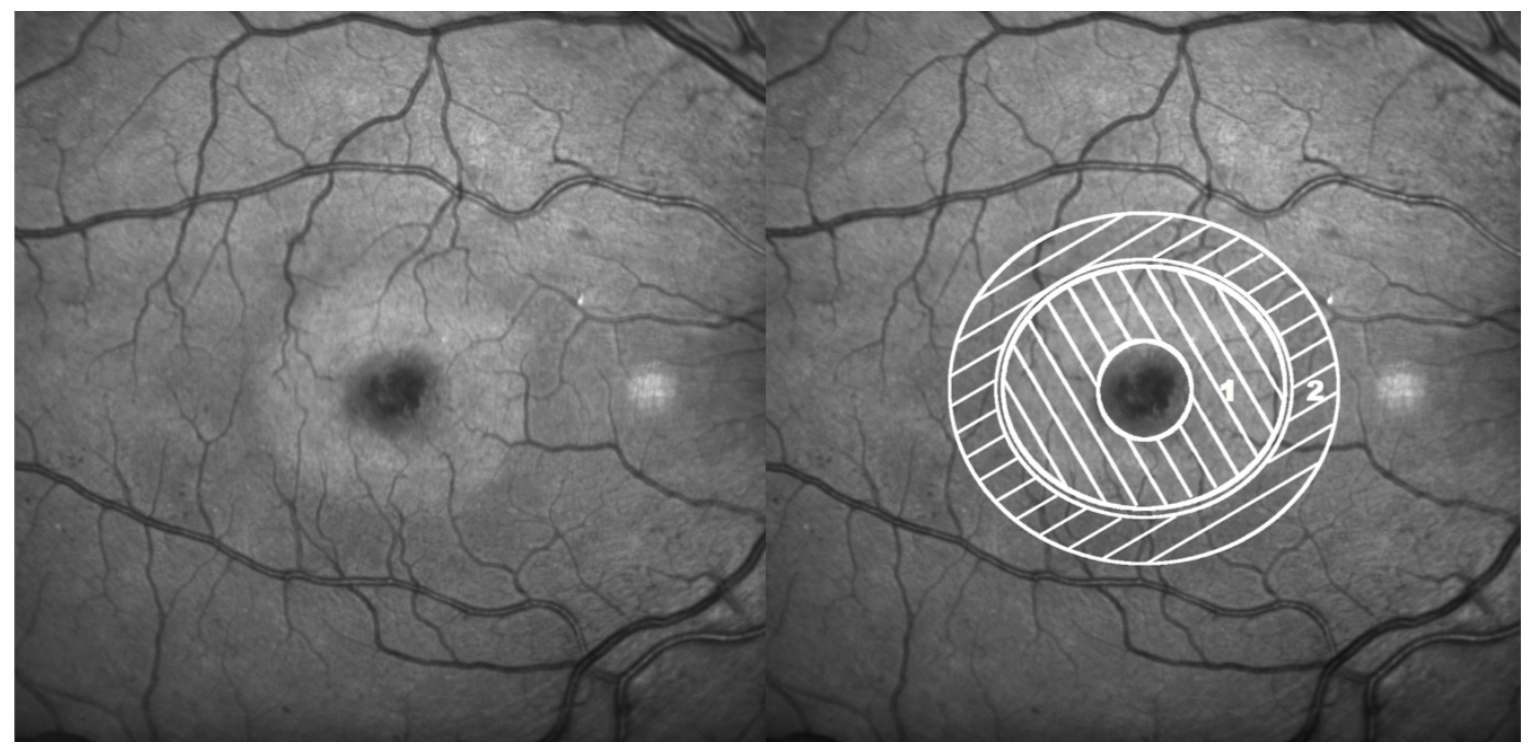


Figure 2.

Example of the effect of bleaching over a sequence of blue light reflectance imaging. The contrast between the parafoveal area and the background is diminished over time. Images in order of sequence: 1-baseline, 5-mid-bleach, 15-last bleached image.

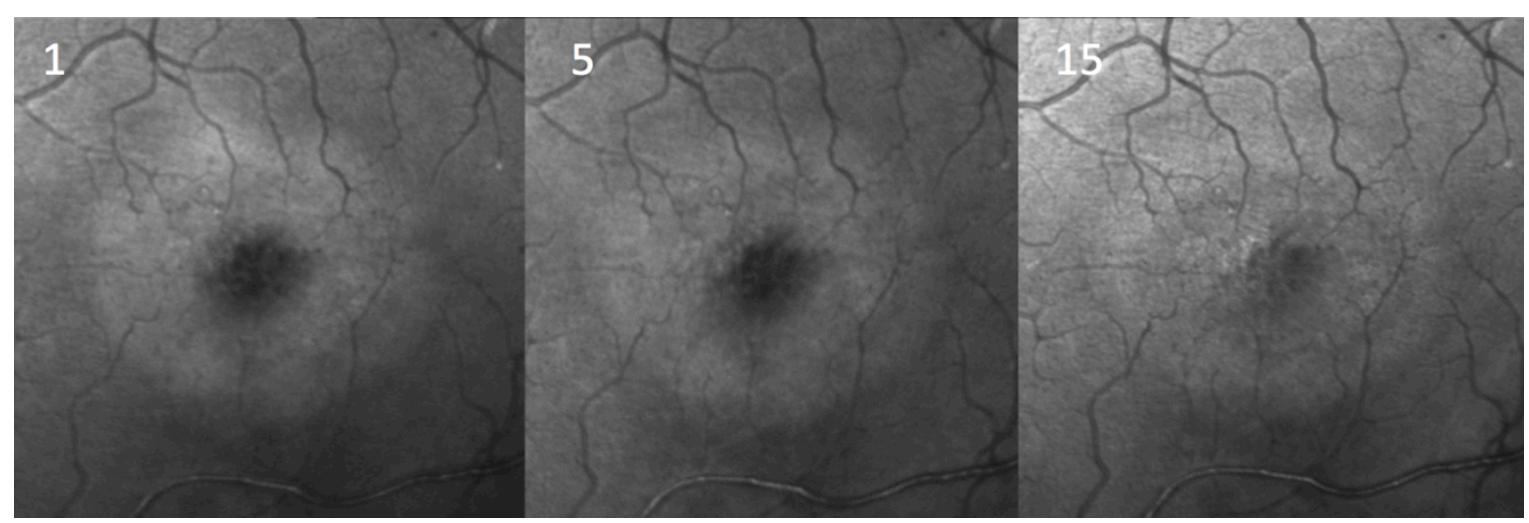


Figure 3.

Effect of bleaching on the difference in blue light reflectance intensity between the abnormal parafoveal area in macular telangiectasia type 2 and the normal paramacular surrounds. Baseline image after 15 minute dark adaptation. Control (circle); MacTel (diamond); with \pm 2 standard error bars.

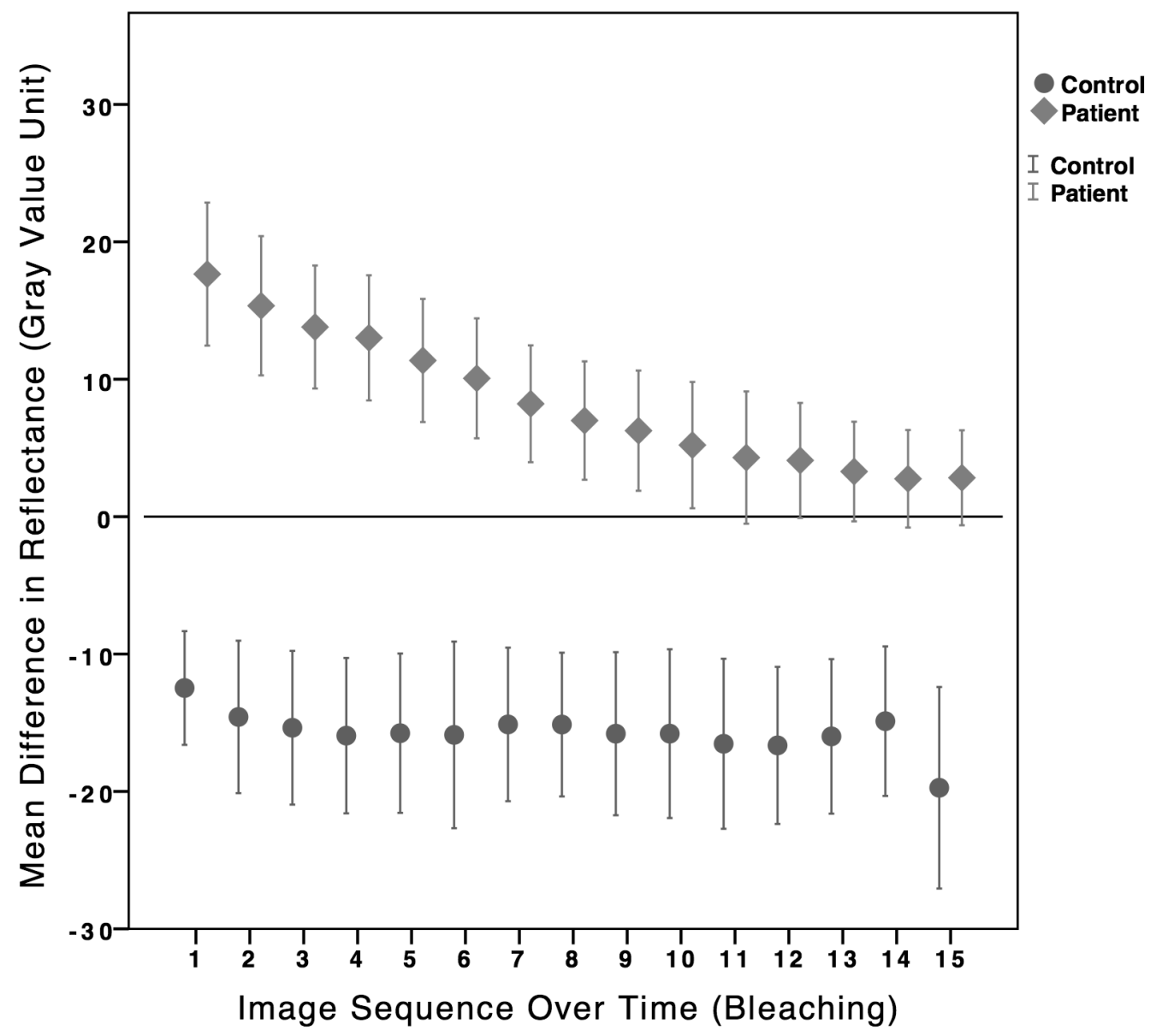


Figure 4.

Example of eyes with macular telangiectasia type 2 who had split imaging performed to selectively bleach the superior half of the fundus. The background paramacular area demonstrates relatively enhanced reflectivity in the superior half (arrow demarcates junction of bleaching) with reduced contrast in the parafoveal region as compared to the inferior half of the fundus.

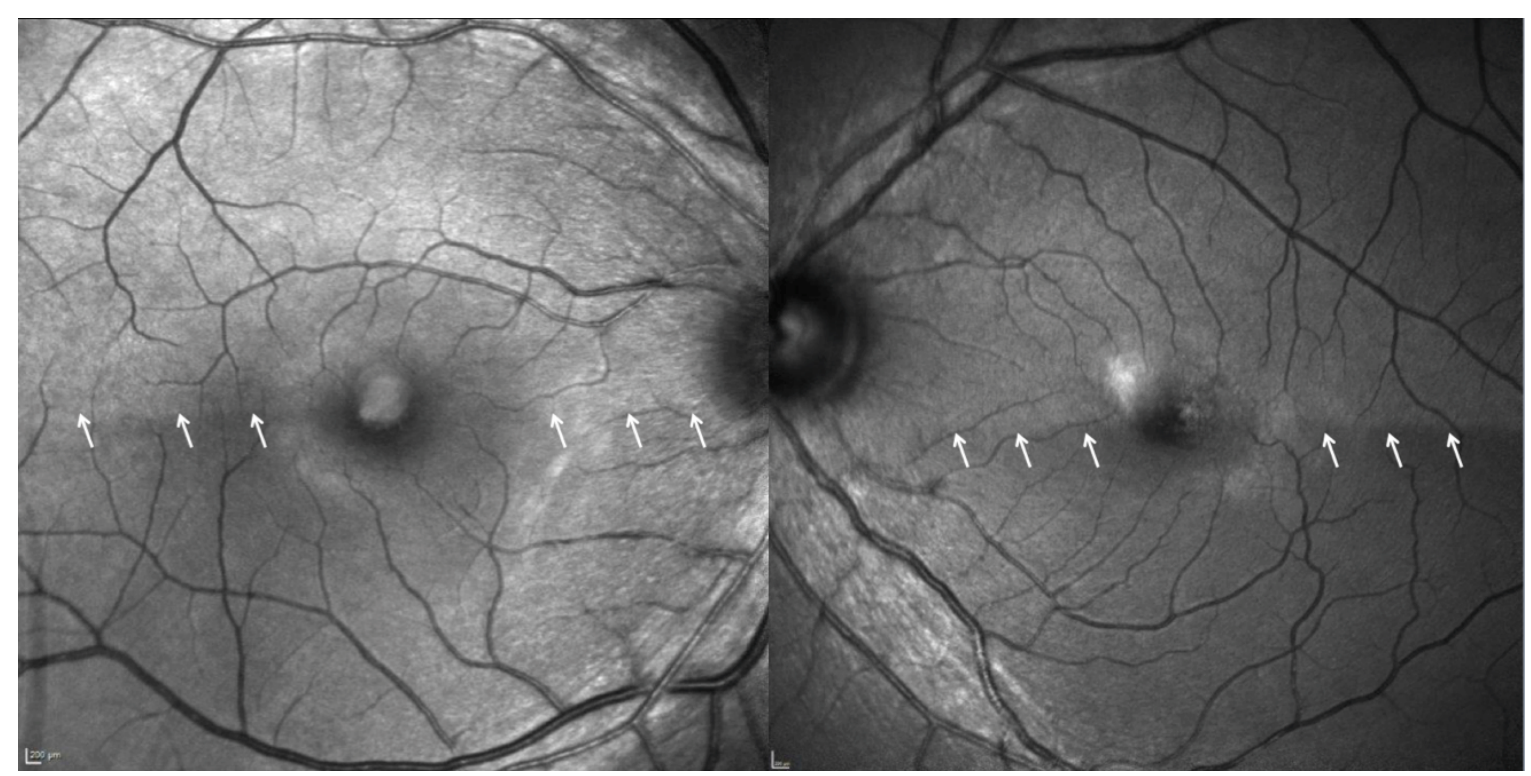




\section{Figure 5.}

Example of bleaching effect with relatively preserved increased reflectance at outer border (arrow) of macular telangiectasia type 2 area compared to more central area. Images in order of sequence: 1-baseline, 5-mid-bleach, 15-last bleached image.

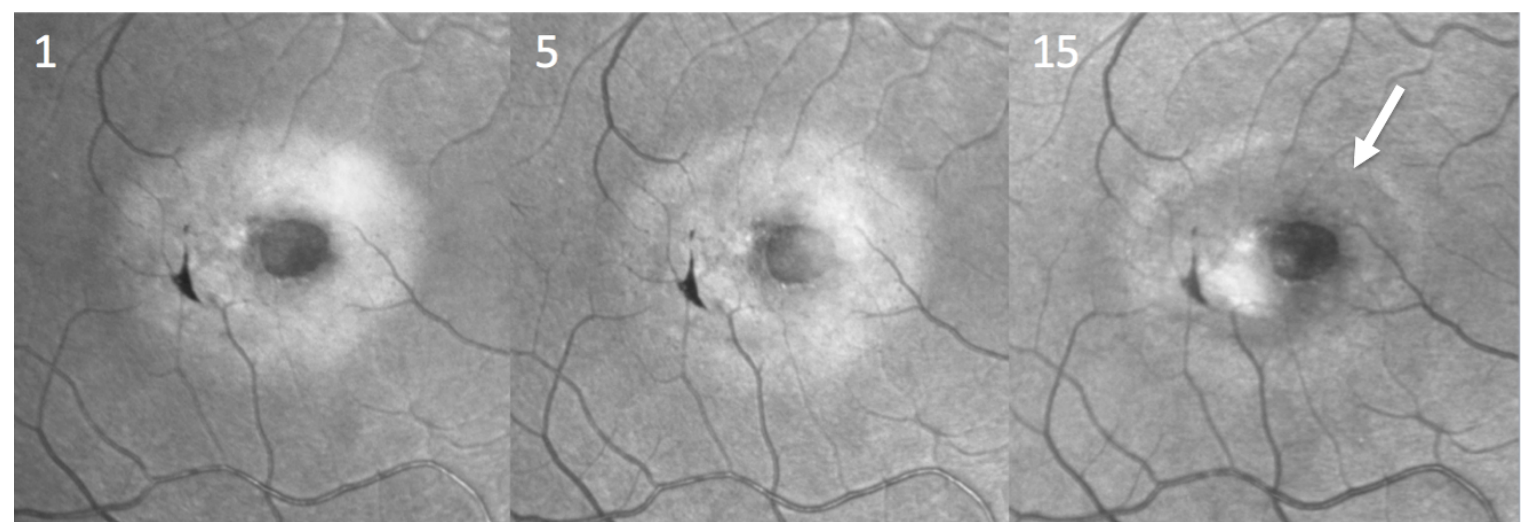


Table 1. BLR imaging grading

\section{Grade Characteristics}

$0 \quad$ Equivocal or absent parafoveal hyper-reflectivity

$1 \quad$ Increased reflectance limited to two temporal quadrants

2A Increased reflectance in outer ring of MacTel area with nasal inferior sparing

2B Increased reflectance of MacTel area with nasal inferior sparing

3A Complete outer ring of increased reflectance in MacTel area

3B Complete increased reflectance of MacTel area 
Table 2. Linear mixed regression model of the effect of time (bleaching) on the difference in signal intensity between the parafoveal versus paramacular surrounds with blue light reflectance imaging.

\begin{tabular}{|c|c|c|c|c|}
\hline \multicolumn{5}{|c|}{ MacTel Patients Versus Controls } \\
\hline & Effect (gray-units) & SE & P-value & \\
\hline Intercept & -15.46 & 5.14 & 0.006 & $*$ \\
\hline MacTel vs. Control & 31.39 & 5.06 & $<0.001$ & $*$ \\
\hline Eye (Right vs. Left) & 1.88 & 3.38 & 0.585 & \\
\hline Effect of Time & -0.22 & 0.33 & 0.516 & \\
\hline Disease status vs. Time & -0.85 & 0.38 & 0.035 & $*$ \\
\hline \multicolumn{5}{|c|}{ Within MacTel Patients Only } \\
\hline & Effect (gray-units) & SE & P-value & \\
\hline Intercept & 15.77600 & 3.26300 & $<0.001$ & * \\
\hline Effect of Time & -1.07300 & 0.20000 & $<0.001$ & $*$ \\
\hline Eye (Right vs. Left) & 2.17900 & 3.84200 & 0.579 & \\
\hline
\end{tabular}

Legend:

SE: Standard error; vs: versus; *: significant p-values $<0.05$ 